\title{
乙酸乙酯的可视化制备
}

\author{
鞠志宇 ${ }^{*}$, 赵书珍，孔灵钰，杨莹芗 \\ 许昌学院化工与材料学院, 河南许昌 461000
}

摘要: 在基础教学实验中引入可视化实验理念以强化酯化反应操作, 对乙酸乙酯的制备实验进行了综合改进。以乙 酸和乙醇为原料, 硫酸氢钠为催化剂、变色硅胶为吸水剂、甲基紫为可视化试剂, 实现了乙酸乙酯可视化制备, 解决 了试剂限购和碳化等问题。气相色谱分析结果表明乙酸几乎完全转化, 并通过氢核磁共振( $\left.{ }^{1} \mathrm{H} N \mathrm{NM}\right)$ 证实了乙酸乙酯 产物结构。利用颜色明显变化进行化学反应过程可视化教学, 让学生在视觉上感知酯化反应的动态过程, 从而增强 学生学习有机化学的兴趣, 活跃学生的思维以及提高学生学习效率。

关键词: 乙酸乙酯; 可视化; 甲基紫; 制备

中图分类号: G64; O6

\section{Visualized Process for Preparing Ethyl Acetate}

\author{
Zhiyu Ju *, Shuzhen Zhao, Lingyu Kong, Yingying Yang \\ School of Chemical \& Material Engineering, Xuchang University, Xuchang 461000, Henan Province, China.
}

\begin{abstract}
In order to introduce the concept of visualized experiment into basic laboratory teaching and to enhance the exercise on esterification, comprehensive improvement was made on the preparation of ethyl acetate. In this experiment, acetic acid reacts with ethanol to produce ethyl acetate in the presence of sodium bisulfate and allochroic silica gel, and the endpoint of the reaction is vidually indicated by methyl violet discoloration. This design of the reaction circumvents the problems of limited reagents and carbonization. The conversion was measured by gas chromatography, and the result showed that acetic acid was almost completely turned into the target product; the structure of the product was confirmed by ${ }^{1} \mathrm{H}$ NMR. This experiment allows students to have a feel of the dynamic process of esterification by the discoloration of methyl violet with the increased acidity of the reaction system. Consequently, it can enhance students' interests in learning organic chemistry, enliven their thinking and improve their study efficiency.
\end{abstract}

Key Words: Ethyl acetate; Visualization; Methyl violet; Synthesis

酯化反应是有机化学中的一类重要反应, 是醇与羧酸反应生成酯和水的反应。酯化反应已经广 泛应用于染料、生物医药、材料等原料的合成 ${ }^{[1]}$ 。典型的酯化反应有乙醇和乙酸的反应, 生成具有芳 香气味的乙酸乙酯, 是常用的有机溶剂、染料和医药的原料。由于其原料价格便宜、易得, 产物具 有一定的香味。因此，乙酸乙酯的制备常被选作本科生有机化学实验的训练项目内容。

教材中的乙酸乙酯的制备实验方法 ${ }^{[2]}$ 存在以下问题: (1) 由于乙醇、乙酸和乙酸乙酯不能使用薄 层色谱分析方法监控反应, 气相色谱监控反应对于本科生基础实验而言, 设备价格昂贵, 监控时间 长(一般需要10分钟/样品)。反应终点监控难, 学生不能有效获得酯化反应过程的感性认知。(2) 以浓

收稿: 2021-10-25; 录用: 2022-01-06; 网络发表: 2022-03-08

“通讯作者, Email: juz16961@163.com

基金资助: 河南省高等教育教学改革研究与实践项目(2019SJGLX404); 河南省高等教育教学改革研究重点项目(2014SJGLX064) 
硫酸为催化剂。它是管制类危险化学品, 购买使用管理严格; 蒸馏反应物时容易碳化、且产生有毒 二氧化硫气体、降低产率, 甚至得不到产物, 严重挫伤学生实验的积极性。为了解决上述问题, 以 实现可视化、绿色化、高效制备乙酸乙酯, 对本实验进行综合改进。

可视化实验是利用颜色、状态、气压等现象的变化, 实现实时、快速呈现反应进度的实验 ${ }^{[3,4]}$ 。 将不能看到或不易检测的实验过程实现直观识别、甚至量化, 使学生能够感知到反应进行的程度。 运用可视化实验, 不仅可以促进学生对实验原理的理解, 还可以改变学生的学习方式, 提高学生进 行科学探究的兴趣。在酯化反应过程中, 随着反应的进行, 乙酸的浓度逐渐降低, 即反应体系中的 $\mathrm{pH}$ 逐渐增大。依据以上分析, 采用合适的酸碱指示剂可以实现实验进度可视化判断, 其可视化实验 的基本原理如图1所示。反应开始时加入一定量的指示剂, 反应体系呈现一种颜色, 随着反应的进行, 反应体系乙酸浓度降低, 酸性减弱, $\mathrm{pH}$ 逐渐增大, 当 $\mathrm{pH}$ 达到一定值后, 颜色发生明显变化, 即反应 达到终点。

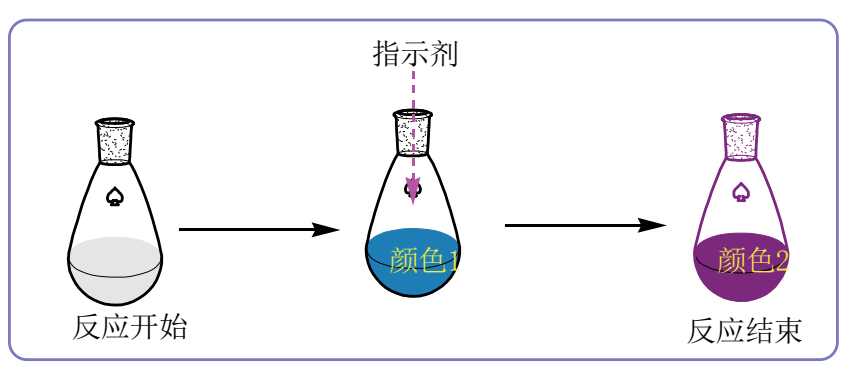

图1 可视化实验的基本原理

另外, 酯化反应若使用浓硫酸作催化剂, 存在容易碳化、且产生有毒二氧化硫气体以及降低产 率等问题, 可能是浓硫酸强的氧化性使乙醇和乙酸碳化造成的, 同时浓硫酸也是管控试剂, 购买和 使用管理严格。为了避免使用浓硫酸, 根据文献 ${ }^{[1]}$ 采用没有氧化性、操作更安全和绿色的硫酸氢钠 作为催化剂。

本文通过对可视化实现条件、催化剂、除水剂及其实验装置的改进进行研究, 实现了乙酸乙酯 的可视化制备。

\section{1 实验部分}

\section{1 反应原理}

乙酸乙酯的制备原理如图2所示。为了提高乙酸乙酯的产率, 本实验采取加入过量乙醇及不断将 反应生成的水通过干燥剂吸收的方法除去, 使反应平衡向着正反应方向移动。催化剂采用硫酸氢 钠 ${ }^{[1]}$, 由于其是强电解质, 可以完全电离, 形成质子, 可以代替浓硫酸起到相同的催化效果。

为了实现反应终点检测的可视化, 采用甲基紫指示剂随酸性变化而发生变色的方法, 其变色原 理如图3所示。甲基紫第一变色范围: $\mathrm{pH} 0.13-0.5$, 颜色由黄色变绿色; 甲基紫第二变色范围: $\mathrm{pH}$ 1.0-1.5, 颜色由绿色变蓝色; 甲基紫第三变色范围: pH 2.0-3.0, 颜色由蓝色变为紫色。由于乙酸乙 酯制备体系随着反应的进行, 乙酸浓度逐渐降低, 酸性由强变弱, 即 $\mathrm{pH}$ 逐步增大。若使用甲基紫监 控其 $\mathrm{pH}$ 变化, 则反应体系颜色由浅蓝色变为紫色。可以肉眼观察到反应体系颜色发生明显变化, 从 而判断反应是否达到终点。

$$
\mathrm{CH}_{3} \mathrm{CO}_{2} \mathrm{H}+\mathrm{CH}_{3} \mathrm{CH}_{2} \mathrm{OH} \underset{\text { Reflux }}{\stackrel{\mathrm{H}^{+}}{\rightleftharpoons}} \mathrm{CH}_{3} \mathrm{COOCH}_{2} \mathrm{CH}_{3}+\mathrm{H}_{2} \mathrm{O}
$$

图2 乙酸乙酯的制备原理 


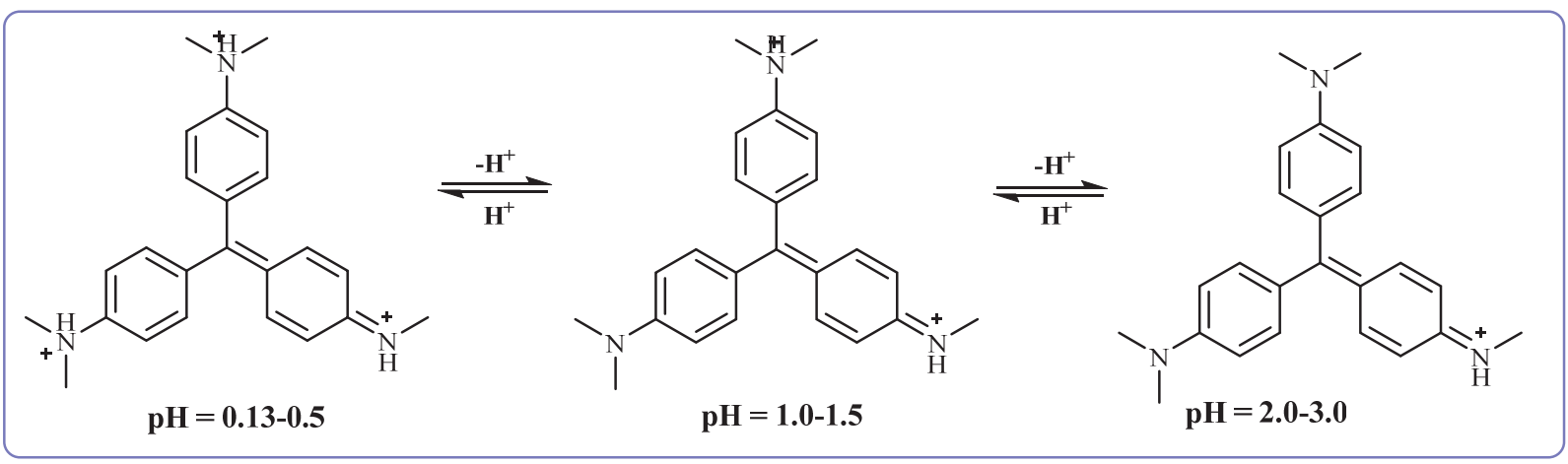

图3 甲基紫变色原理

\section{2 实验仪器与试剂}

冰醋酸、无水氯化钲, AR, 天津市科密欧化学试剂有限公司; 无水乙醇、碳酸钠, AR, 天津市 富宇精细化工有限公司; 硫酸氢钠、无水硫酸镁, AR, 北京化工厂; 甲基紫, AR, 天津北辰方正化 学试剂厂; 甲基橙、甲基红, AR, 天津大茂化学试剂厂; 变色硅胶, 上海昌全硅胶干燥剂有限公司; 无水硫酸铜、无水氯化钴, 福晨化学试剂有限公司; 分子篮, 江西金陶科技股份有限公司。去离子 水为学校自制。

核磁共振仪: Bruker Ascend ${ }^{\mathrm{TM}}{ }^{1} \mathrm{H} N M R 400 \mathrm{MHz}, \mathrm{CDCl}_{3}$ 为溶剂, TMS 为内标。气相色谱: 赛默 飞TRACE1300, 氢火焰离子化检测器(FID), 采取内标法。色谱条件: 色谱柱为EN-20气相毛细色谱 柱, 气化室温度为 $150^{\circ} \mathrm{C}$, 柱温为 $120^{\circ} \mathrm{C}$, 检测器温度为 $180^{\circ} \mathrm{C}$, 流速: 氮气 $0.1 \mathrm{MPa}$ 、氢气 $0.1 \mathrm{MPa} 、$ 空气 $0.1 \mathrm{MPa}$ 。

\section{3 实验步骤}

在 $50 \mathrm{~mL}$ 圆底烧瓶中加入 $5.8 \mathrm{~mL}$ 酸和 $9.1 \mathrm{~mL}$ 乙醇, 搅拌状态下加入 $1.4 \mathrm{~g}$ 硫酸氢钠, 以及4-6滴 $1 \%$ 的甲基紫指示剂(乙醇为溶剂配制), 溶液由无色变为蓝色; 在小孔冷凝柱中加入 $3.5 \mathrm{~g}$ 变色硅胶, 然后依次将其装置和直型冷凝管安装在烧瓶上, 加热至回流50 min, 观察反应体系溶液由蓝色变为 紫色, 停止反应, 得粗乙酸乙酯。稍冷后, 缓慢向烧瓶中加入饱和碳酸钠水溶液, 至不再有二氧化 碳气体逸出, 有机相用 $\mathrm{pH}$ 试纸检测显示中性。将液体转入分液漏斗中, 摇振后静置, 分去水相, 有 机相用 $12 \mathrm{~mL}$ 饱和食盐水洗涤后, 再每次用 $4 \mathrm{~mL}$ 饱和氯化钲溶液洗涤两次。弃去下层液, 酯层用无 水硫酸镁干燥。将干燥后的粗乙酸乙酯滤入 $25 \mathrm{~mL}$ 蒸馏瓶中进行蒸馏, 收集 $73-78^{\circ} \mathrm{C}$ 馏分, 得到无色 液体, 质量为 $6.4 \mathrm{~g}$ 。

\section{2 结果与讨论}

\section{1 实验进程可视化条件探讨}

为了探讨实验进程的可视化条件, 分别选择甲基紫、甲基橙和甲基红为指示剂, 在 $50 \mathrm{~mL}$ 圆底烧 瓶中加入 $5.8 \mathrm{~mL}$ 乙酸和 $9.1 \mathrm{~mL}$ 醇, 搅拌状态下加入 $1.4 \mathrm{~g}$ 硫酸氢钠, 分别滴加 $4-6$ 滴 $1 \%$ 的甲基紫, 甲基橙以及甲基红, 进行平行实验。实验结果表明: 随着反应时间的延长, 甲基橙和甲基红反应体 系无明显颜色变化; 而甲基紫反应体系颜色变化明显, 反应开始, 体系颜色为图4(a)显示颜色, 反应 大约 $20 \mathrm{~min}$ 后, 反应体系颜色呈现为图4(b)颜色, 反应时间约 $50 \mathrm{~min}$ 时, 反应体系颜色变为图4(c)颜 色, 说明甲基紫对反应终点具有指示作用。为了进一步验证甲基紫指示反应终点的可能性, 当反应 体系颜色变为图4(c)颜色时, 通过气相色谱法跟踪反应物转化情况, 检测结果表明乙酸的转化率为 $92.49 \%$, 说明乙酸几乎完全转化。反应产物经过纯化并通过 ${ }^{1} \mathrm{H} N \mathrm{NR}$ 确认了其结构。因此, 甲基紫为 最佳可视化指示剂。 


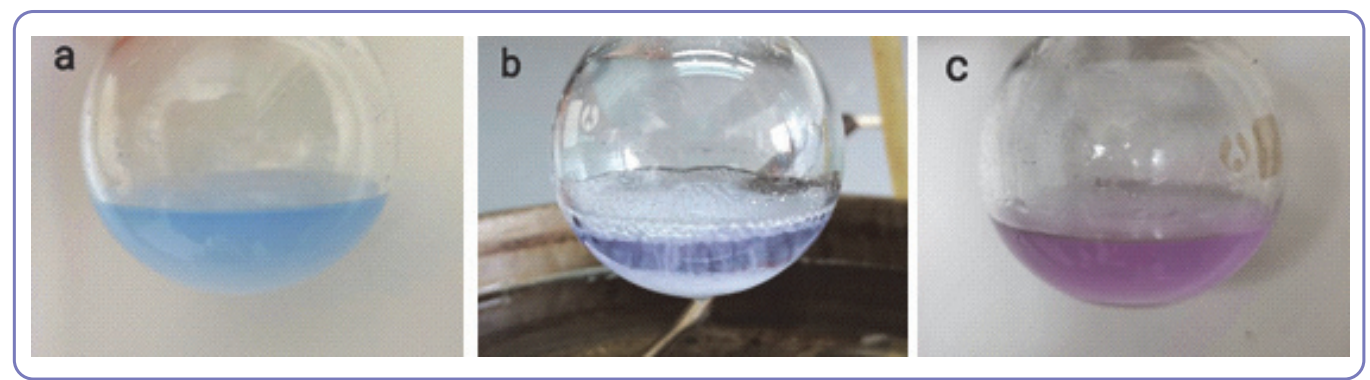

图4 甲基紫指示的反应进程颜色变化情况

(a) 反应前颜色; (b) 反应中颜色; (c) 反应后颜色 电子版为彩图

\section{2 除水剂的选择}

为了探讨除去反应生成水的条件, 分别选择无水硫酸铜、无水氯化钴、变色硅胶和分子篎为吸 水剂。并分别将 $3.5 \mathrm{~g}$ 变色硅胶、分子篎、无水硫酸铜和无水氯化钴装入小孔冷凝管中, 在 $50 \mathrm{~mL}$ 圆底 烧瓶中加入 $5.8 \mathrm{~mL}$ 乙酸和 $9.1 \mathrm{~mL}$ 乙醇, 搅拌状态下加入 $1.4 \mathrm{~g}$ 硫酸氢钠, 分别滴加 $4-6$ 滴 $1 \%$ 的甲基紫, 加热至回流50 min。实验结果表明: 无水氯化钴为吸水剂时, 很快随着溶剂流入反应体系中, 导致 可视化失败; 无水硫酸铜和分子篮为吸水剂时, 转化率低于变色硅胶, 同时存在堵塞液体回流的问 题, 不能充分反应和有效吸收水分。使用变色硅胶做吸水剂时, 反应前, 硅胶呈现深蓝色(图5左), 随着反应的进行, 变色硅胶不断吸水, 自身的颜色逐渐变浅, 当反应结束后, 硅胶表面出现浅紫色 (图5右)。由此说明，硅胶不仅具有吸收水的作用，还具有可视化的效果。

\section{3 实验装置的改进}

本实验为了提高乙酸乙酯的产率, 传统采用蒸馏的方法移除生成的水。若采用变色硅胶直接加 入反应体系, 实验结果表明: 严重影响甲基紫指示反应进程, 不能实现可视化。因此对实验装置进 行了改进, 运用固定床反应器原理 ${ }^{[5]}$ 自行设计并定制了小孔冷凝管, 其结构如图6所示, 因其具有承 载板、小孔和管状结构, 从而实现承载吸水剂、吸水反应、冷凝和固液分离的功能。

\section{4 催化剂的选择}

为了解决浓硫酸购买管制和容易碳化问题, 选取了文献报道 ${ }^{[1,6,7]}$ 的硫酸氢钠、磷铇酸、硅钨酸、 氯化铝等几种催化剂, 将 $3.5 \mathrm{~g}$ 变色硅胶装入小孔冷凝管中, 在 $50 \mathrm{~mL}$ 圆底烧瓶中加入 $5.8 \mathrm{~mL}$ 酸和 $9.1 \mathrm{~mL}$ 乙醇, 搅拌状态下加入 $10 \%$ 的不同催化剂, 滴加 4-6滴 $1 \%$ 的甲基紫, 加热至回流 $50 \mathrm{~min}$ 。气相

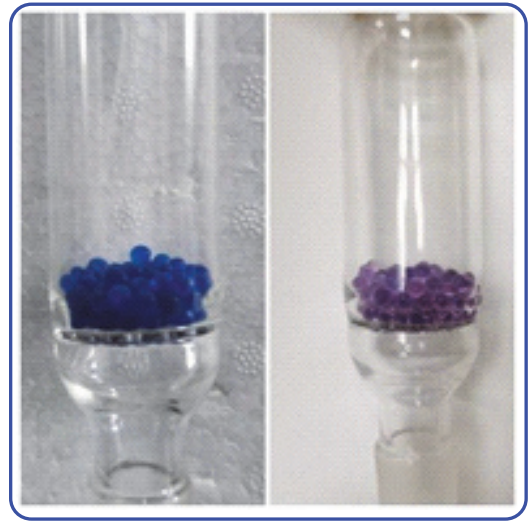

图5 变色硅胶反应前后对比 电子版为彩图

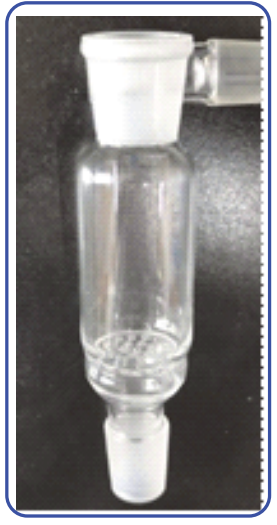

图6 小孔冷凝管的结构图 
检测结果表明, 转化率分别为: $92.49 \% 、 84.59 \%$ 、87.12\%和 $75.36 \%$ 。由于氯化铝易水解, 操作和可 视化难度大, 转化率也最低; 虽然磷铇酸和硅铇酸与硫酸氢钠催化效果基本接近, 但是磷钨酸和硅 钨酸分子量大, 用量多, 存在固废后处理和价格较贵问题。因硫酸氢钠具有操作安全、价格便宜、 环保的特点, 不仅解决了试剂购买不受管制, 还解决了碳化问题, 因此, 最终选取硫酸氢钠为催化 剂。

\section{3 结语}

本实验以直观、可视化实验为手段, 以激发学生学习有机化学的兴趣为指导思路, 为了探索乙 酸乙酯制备终点可视化监控条件、解决酯化反应中碳化和提高酯化率问题, 进行了一系列实验探究。 研究结果表明, 甲基紫为乙酸乙酯制备可视化监控最佳指示剂, 变色硅胶为吸水剂, 转化率为 $92.49 \%$ 。可视化实验帮助学生加深了对可逆反应过程和固定床反应器原理的理解, 达到了预期的实 验目的。同时运用所学知识解决实际问题, 培养学生应用能力, 激发学生做实验的兴趣。

通过实验证明加入指示剂和改进装置在乙酸乙酯制备实验中具有可行性、安全性和高效性。乙 酸乙酯可视化制备实验是为了更加直观地观察到乙酸乙酯的生成过程, 是有目的地引导学生观察实 验过程中合成酯的进度, 认识酯化反应, 培养学生应用所学知识发现、分析和解决具体问题的能力 及实事求是的科学态度。改进后的乙酸乙酯可视化制备实验具有教学直观性、实用性、可操作性和 科学性, 符合本科生实验教学的要求。

\section{参 考 文 献}

[1] 迟卫军. 化学与黏合, 2007, 29(6), 451.

[2] 王清廉, 李瀛. 有机化学实验. 第4版. 北京: 高等教育出版社, 2016.

[3] 刘江, 王丽娇, 双伸苹. 化学教育(中英文), 2019, 40 (8), 51 .

[4] 高昆. 学理论, 2014, 14 (2), 180.

[5] 杨玉峰. 化学教学, 2019, No. 3, 67.

[6] 陈平, 陈宁一, 邵全省. 化学教学, 1987, No. 1, 24.

[7] 王秀丽, 李兴伟, 蔡艳荣, 黄宏志. 锦州师范学院学报(自然科学版), 2001, No. 3, 26. 